\title{
Histopathological evaluation of renal allograft biopsies in Nepal: interpretation and significance
}

\author{
Aryal $\mathrm{G}^{1}$, Shah $\mathrm{DS}^{2}$ \\ ${ }^{1}$ Department of Pathology, KIST Medical College and Teaching Hospital, Lalitpur, Nepal \\ ${ }^{2}$ Nephrology Unit, Department of Medicine, Tribhuvan University Teaching Hospital, Kathmandu, Nepal
}

\section{Keywords: \\ Kidney transplantation; Allograft; \\ Biopsy; \\ End -stage renal disease; \\ Rejection}

\begin{abstract}
Background: Renal allograft biopsy remains the gold standard for the diagnosis of graft dysfunction. Protocol renal allograft biopsies have provided insights into pathogenesis of early and late allograft injury and guided clinical management. The aim of this study was to find out the causes of early and late graft dysfunction in renal allograft biopsies taken at different time points after transplantation.
\end{abstract}

Materials and Methods: Between August 2008 and February 2011, a total of 98 renal allograft biopsies from 62 kidney transplants recipients were received in the department of Pathology, KIST Medical College and Teaching Hospital. Renal allograft biopsies were interpreted according to the Schemes of Banff '09 update.

Results: The maximum numbers of recipients were in the age group of 31-40 years. Age ranges from 16 to 61 years. Out of 62 recipients, there were $47(75.8 \%)$ males and $15(24.2 \%)$ females. The time of biopsy ranges from 0 hour to 6 years after transplantation. There were $9(9.18 \%)$ cases of acute T cell-mediated rejection, $8(8.16 \%)$ cases of acute antibody-mediated rejection, $6(6.12 \%)$ cases of acute ischemic injury, $6(6.12 \%)$ cases of borderline lesions and $4(4.08 \%)$ cases of chronic calcineurin inhibitor toxicity.

Conclusion: In the early post-transplant period (0-6 months), acute antibody-mediated rejection, acute ischemic injury and $\mathrm{T}$ cell-mediated rejection are the major causes for graft dysfunction. In the late post transplant period (> 6 months after transplant), transplant glomerulopathy, chronic calcineurin inhibitor toxicity, viral infections and graft senescence including donor derived disease result in poor late graft survival.

\section{INTRODUCTION}

Renal transplantation is safe and cost effective and markedly improves survival and quality of life for patients with end-stage renal disease (ESRD). On August 2008, the

\author{
Correspondence: \\ Dr. Gopi Aryal, $M D, P h D$ \\ Associate Professor, Department of Pathology, KIST Medical College and \\ Teaching Hospital, Lalitpur, Nepal \\ GPO Box: 11442 \\ E-mail: gopiaryal@hotmail.com
}

first successful renal transplantation was done in Nepal with the help of the Australian surgeon, Dr David Francis. ${ }^{1}$ Since then, more than 100 successful renal transplants have been performed at Tribhuvan University Teaching Hospital. Renal transplantations also started at Bir Hospital, Kathmandu in December 2008 by a team of Nepalese surgeons.

Before 2008, Nepalese people used to go to India for renal transplantation and the major source of kidneys were from living unrelated kidney donors. ${ }^{2}$ 
Renal allograft biopsy remains the gold standard for the diagnosis of graft dysfunction. ${ }^{3}$ It plays the major role in the diagnosis and subsequent therapy of allograft dysfunction. Rejection is most important, but is only one of many causes of allograft dysfunction. The other causes of graft dysfunction are acute ischemic injury, calcineurin inhibitor (CNI) toxicity, infections, including pyelonephritis and polyomavirus nephropathy, chronic obstruction/reflux, hypertension, and recurrent and de novo disease.

The degree of Human Leukocyte Antigen (HLA) mismatch and the presence of preexisting (sensitized) or de-novo HLA antibodies, particularly donor specific antibodies (DSAs) are important determinants of graft survival. ${ }^{4}$ Until the early 1990 s, rejection of renal allograft was classified into hyperacute (minutes to hours, due to preformed DSAs), accelerated (within first two transplant weeks), acute (weeks to months) and chronic (months to years) rejection.

There was no standardized international diagnostic category for renal allograft biopsies resulting in considerable heterogeneity in reporting among the various centers. In 1991, a group of renal pathologists, nephrologists, and transplant surgeons formulated a schema for nomenclature and classification of renal allograft biopsies in Banff, Alberta, Canada. ${ }^{5}$ For the last 20 years, Banff classification of renal allograft pathology has grown to be the major standard classification in renal transplant pathology and in international clinical trials of new antirejection agents. The original Banff classification remains updated at regular interval, the latest being in August 2009. ${ }^{6}$ The most significant change in 2009 compared to 2007 Banff schema $^{7}$ was the acceptance and definition of antibody- mediated rejection (ABMR).

There are many causes of early graft dysfunction. Immediately after transplant, ischemic injury is the major concern, while acute rejection can manifest usually after the first week. The key histological feature of acute rejection is the presence of inflammation that targets renal tubules, vessels and glomeruli. ABMR is less easily diagnosed on histological criteria alone. It must be considered largely in the pre-sensitized patient. In renal biopsy, complement split product $\mathrm{C} 4 \mathrm{~d}$ deposition in the peritubular capillaries is considered to be a marker for ABMR. ${ }^{8}$

Acute T cell-mediated rejection (TCMR) arises because of recipient immune activation by donor antigens and occurs mostly in the context of mismatched HLA. Histologically, the biopsy shows mononuclear cell infiltration within the interstitium, tubules and arterial endothelium. Inflammation must involve at least $25 \%$ of the interstitium with moderate tubulitis to diagnose rejection. A higher grade is applied if there is severe tubulitis. Glomeruli appear normal in the majority of the cases of acute cellular rejection.

Late graft dysfunction occurs months to years post- transplantation and is multifactorial, involving both immunological and non-immunological factors. Immunological factors include the degree of HLA matching, whether the recipient has previously been sensitized to HLA with the development of donor specific HLA antibodies, and the effectiveness of immunosuppressant used. The nonimmunological factors include recipient age and general health, donor type and original cause of renal failure. Chronic CNI toxicity and hypertension are major causes of late graft loss. ${ }^{9}$ Chronic humoral rejection has now been defined in the form of transplant glomerulopathy (TG), which manifests clinically around 5 years after transplant. ${ }^{10,11}$

The morphological sequelae of chronic rejection are now established, and the various other causes have also been better defined. With attention being paid to identifying specific causes, the term "chronic allograft nephropathy (CAN)" has been abandoned and, in the absence of a specific cause, the recommended term is interstitial fibrosis and tubular atrophy, not otherwise specified (IF/TA, NOS) ${ }^{12}$

Protocol renal allograft biopsies at fixed time points from transplantation provide insights into the pathogenesis of early and late allograft injury. ${ }^{13-15}$ It helps to detect subclinical rejection, which is mostly seen during the first month of transplantation and it is associated with the progression to IF/TA. ${ }^{16}$

The aim of this study was to find out the causes of early and late graft dysfunction in renal allograft biopsies taken at different time points after transplantation in one of the university hospital in Nepal.

\section{MATERIALS AND METHODS}

Between August 2008 and February 2011, a total of 98 renal allograft biopsies from 62 kidney transplants recipients were received in the department of Pathology, KIST Medical College and Teaching Hospital. All the biopsies were done in Tribhuvan University teaching Hospital, Kathmandu, Nepal. Implantation biopsy (0-hour) was taken after vascular anastomosis and before wound closure.

Biopsy specimens prepared for light microscopy were formalin-fixed and paraffin-embedded. The sections were cut at $3-4 \mu$ thickness. Two slides were stained with Hematoxylin and eosin (HE), two with Periodic Acid-Schiff (PAS) and one slide with Masson Trichrome stain. All the biopsies were interpreted by author. Optimal biopsy was defined as a specimen that contains at least 10 glomeruli and 2 arteries; a marginal biopsy having 7 to 9 glomeruli and 1 artery. ${ }^{17}$ The presence of 7 glomeruli and 1 artery was considered minimally acceptable. The sections with fewer than 7 glomeruli or no arteries or with only medulla were considered non-diagnostic specimen. The relevant clinical history like rapid rise in serum creatinine, degree of sensitization, anti-HLA antibody status, previous infection 
and allograft age were retrieved from patient record file or by telephone communication with consulting nephrologist.

Histological diagnosis was made according to the Banff '09 update $^{6}$ as shown in Table 1 . This semiquantitative scorings system is used for the classification and grading of shortand long-term changes that occur in the glomeruli, tubules, interstitium, arteries, and arterioles of a renal transplant. Scores range from 0 to 3 , with higher scores indicating more severe abnormalities. Statistical analysis was done by using Statistical Package for the Social Sciences 17.0 software.

\section{RESULTS}

Of 98 biopsy specimens from 62 recipients, 9 were nondiagnostic because of insufficient tissue for evaluation. There were total of 54 protocols (0-hour) and 44 clinically indicated biopsies which were performed at or near the times indicated in the recommended protocol. ${ }^{18}$

Histopathological findings of 98 renal allograft biopsies are shown in Table 2 . There were $9(9.18 \%)$ cases of TCMR, 8 $(8.16 \%)$ cases of ABMR, $6(6.12 \%)$ cases of acute ischemic

Table 1: Banff 97 diagnostic categories for renal allograft biopsies-Banff '09 update

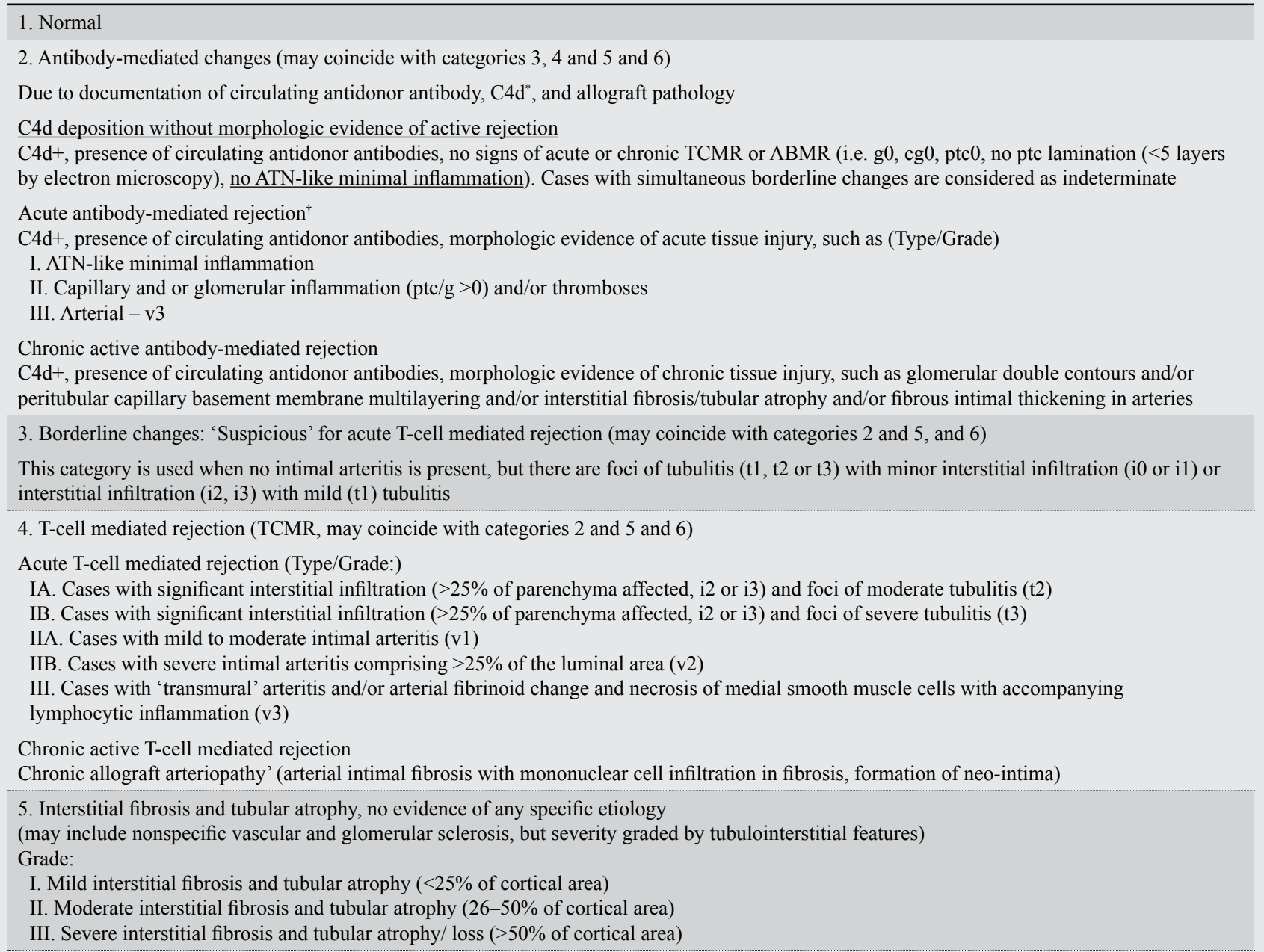

6. Other: Changes not considered to be due to rejection- acute and/or chronic; may include isolated g, cg, or cv lesions and coincide with categories $2,3,4$, and 5

ATN, acute tubular necrosis

The 2009 updates are underlined. All existing scoring categories (g, t, v, i, ptc, cg, ct, ci, cv, ah, mm) remain unchanged ${ }^{7,17}$

${ }^{*}$ Please refer to Banff 2007 classification paper ${ }^{7}$

${ }^{\dagger}$ Suspicious for antibody-mediated rejection if $\mathrm{C} 4 \mathrm{~d}$ (in the presence of antibody) or alloantibody (C4d+) not demonstrated in the presence of morphologic evidence of tissue injury. 
injury, $6(6.12 \%)$ cases of borderline changes and 4 cases (4.08\%) of chronic CNI toxicity. In biopsy specimens obtained at implantation ( 0 -hour biopsy, $\mathrm{n}=54$ ), 37 biopsies were essentially normal and 6 showed acute ischemic changes.

The time of biopsy ranged from 0 hour to 6 years after transplantation. The maximum number of biopsy specimens obtained was 4 per recipient (range 0 to 4 ). A total of 9 biopsy specimens were obtained at 1 week, 7 at 4 weeks, 12 at 3 months, 8 at 6 months, 5 at 1 year, and 3 at 6 years. The recipient of post transplantation biopsy at 6 years had undergone renal transplantation twice in India. The timing of biopsy and corresponding histopathological findings are shown in Table 3.

Out of 62 recipients, there were $47(75.8 \%)$ males and 15 $(24.2 \%)$ females. The maximum numbers of recipients were in the age group of 31-40 years $(19 / 62,30.65 \%)$. Age ranged from 16 to 61 years. In the age group of 10-20 years, majority of the biopsies were normal (6/9, 96.67\%). There were $58.33 \%, 37.03 \%, 42.85 \%$ and $63.6 \%$ of normal biopsies in the age group of 21-30 years, 31-40 years, 41-50 years and 51-60 years of age group respectively.

\section{DISCUSSION}

Chronic kidney disease leading to ESRD has been increasing worldwide. Based on the global scenario, ESRD incidence in Nepal is estimated to be approximately 2600 per year. ${ }^{19}$ There are 3 methods of renal replacement therapy available to patients: continuous ambulatory peritoneal dialysis, hemodialysis and renal transplantation. Of these transplantation is the most cost effective and provides better quality of life. Nepal performed its first successful kidney transplant in 2008. ${ }^{1}$ Renal allograft biopsy is the most sensitive diagnostic tool for determining the cause of graft dysfunction and for the diagnosis of allograft rejection. ${ }^{3}$
Out of 98 renal allograft biopsies, there were $9(9.18 \%)$ cases of TCMR, $8(8.16 \%$,) cases of ABMR, $6(6.12 \%)$ cases of acute ischemic injury, $6(6.12 \%)$ cases of borderline changes and 4 cases $(4.08 \%)$ of chronic CNI toxicity.

Acute ischemic tubular injury (such as with delayed graft function), acute rejection and acute CNI toxicity are common causes of acute allograft dysfunction, whereas chronic rejection, chronic CNI toxicity, hypertension, chronic infection/obstruction and donor-derived disease contribute to late allograft dysfunction. Acute ischemic injury is identified by the typical changes of tubular degeneration and regeneration. Inflammatory cells are usually located adjacent to severely damaged tubules. It shows dilated and thinned tubules with epithelial simplification and loss of PAS positive brush border (fig. 1). Luminal sloughed out cells and cellular debris are seen. The interstitium is edematous with a mild mononuclear cellular infiltrate and areas of hemorrhage. Prominently dilated tubules suggest obstruction, but require correlation with imaging studies.

\begin{tabular}{|c|c|c|}
\hline Diagnosis & Number of biopsy & Percentage \\
\hline Normal & 50 & 51.04 \\
\hline Acute ischemic injury & 6 & 6.12 \\
\hline Borderline changes & 6 & 6.12 \\
\hline T-cell mediated rejection & 9 & 9.18 \\
\hline Antibody-mediated rejection & 8 & 8.16 \\
\hline Calcineurin inhibitor toxicity & 4 & 4.08 \\
\hline Acute interstitial nephritis & 3 & 3.06 \\
\hline Transplant glomerulopathy & 1 & 1.02 \\
\hline Viral infection & 1 & 1.02 \\
\hline IF/TA, NOS* & 1 & 1.02 \\
\hline Non-diagnostic & 9 & 9.18 \\
\hline Total & 98 & 100.0 \\
\hline
\end{tabular}

Table 3: Histopathological findings based on time at biopsy

\begin{tabular}{|c|c|c|c|c|c|c|c|c|c|c|c|c|}
\hline \multirow[b]{2}{*}{ Timing of biopsy } & \multicolumn{11}{|c|}{ Diagnosis } & \multirow[b]{2}{*}{ Total } \\
\hline & Normal & $\begin{array}{l}\text { Acute } \\
\text { ischemic } \\
\text { injury }\end{array}$ & $\begin{array}{l}\text { Borderline } \\
\text { changes }\end{array}$ & ABMR & TCMR & AIN & $\mathrm{CNI}$ & TG & IF/TA & Viral & $\begin{array}{c}\text { Non- } \\
\text { diagnostic }\end{array}$ & \\
\hline 0 time & 37 & 6 & 1 & 0 & 1 & 0 & 0 & 0 & 1 & - & 8 & 54 \\
\hline Within a week & 3 & - & 0 & 3 & 3 & 0 & 0 & 0 & - & - & 0 & 9 \\
\hline 2-4 weeks & 2 & - & 1 & 4 & 0 & 0 & 0 & 0 & - & - & 0 & 7 \\
\hline 2-3 months & 4 & - & 1 & 1 & 2 & 2 & 2 & 0 & - & - & 0 & 12 \\
\hline 4-6 months & 3 & - & 1 & 0 & 2 & 0 & 0 & 1 & - & - & 1 & 8 \\
\hline $7-12$ months & 1 & - & 0 & 0 & 1 & 1 & 1 & 0 & - & 1 & 0 & 5 \\
\hline$>12$ months & 0 & - & 2 & 0 & 0 & 0 & 1 & 0 & - & - & 0 & 3 \\
\hline Total & 50 & 6 & 6 & 8 & 9 & 3 & 4 & 1 & 1 & 1 & 9 & 98 \\
\hline
\end{tabular}




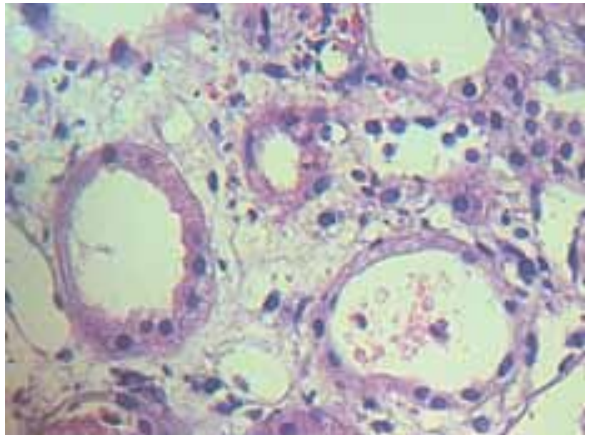

Figure 1: Dilated tubules with epithelial simplification and loss of PAS positive brush border. Luminal sloughed out cells and fragmented red cells are seen within tubular lumina. The interstitium is edematous with areas of hemorrhage (PAS stain, X400).

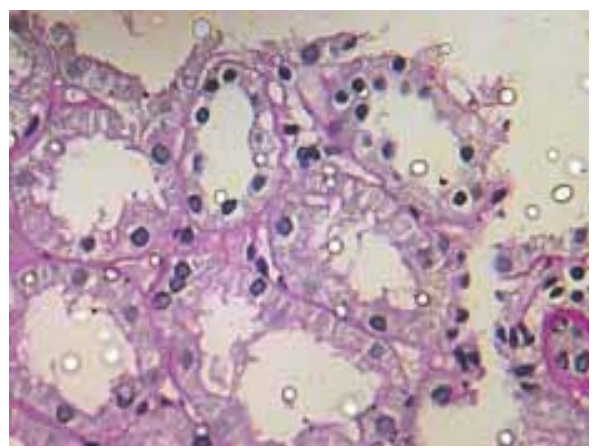

Figure 3A: Banff type 1 TCMR is characterized by tubulitis lesion showing lymphocytes within the tubular basement membrane and between epithelial cell nuclei. It is seen as a dark blue nucleus, with a clear halo, contrasting with the less dense and larger nucleus of the tubular epithelium nuclei (PAS stain, X400).

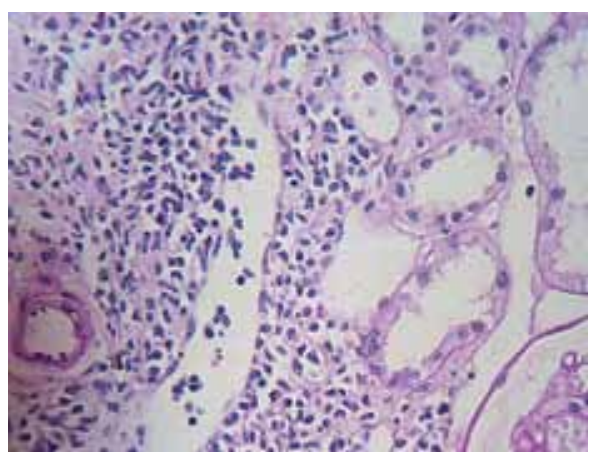

Figure 4A: ABMR. Dilated peritubular capillaries show aggregates of mononuclear inflammatory cells (ptc3). Neutrophil is evident within tubular lumen. (PAS stain, X400).

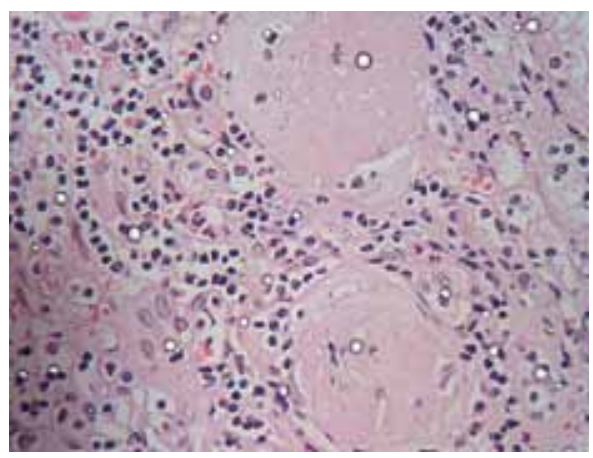

Figure 5A: Acute tubulointerstitial nephritis showing an abundant neutrophilic infiltrate and tubular micro-abscesses (HE stain, X40).

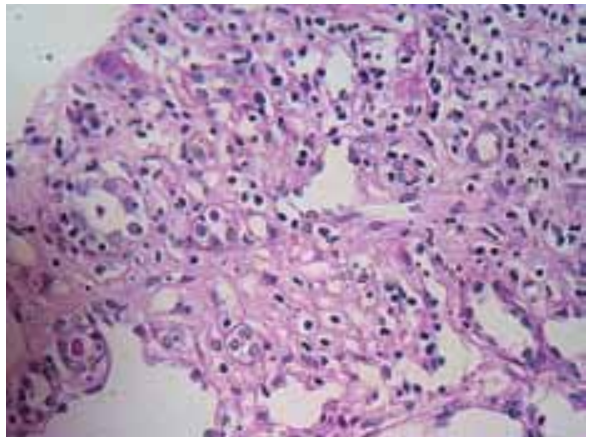

Figure 2: Borderline changes. There are foci of tubulitis ( $t 1)$ with minor interstitial infiltration of mononuclear cells (PAS stain, X400).

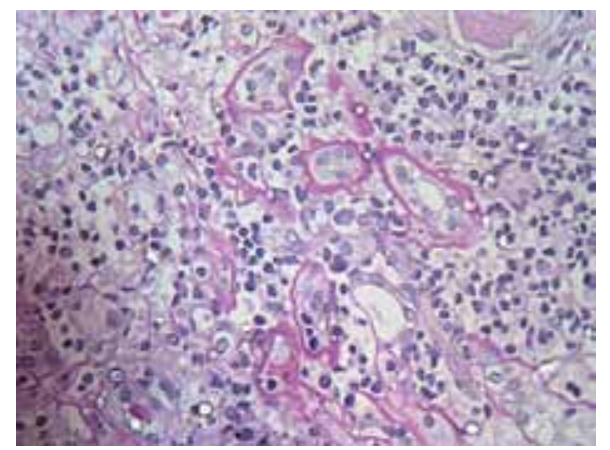

Figure 3B: Banff type 1 TCMR is characterized by significant interstitial infiltration by mononuclear cells and foci of moderate tubulitis (PAS stain, X400).

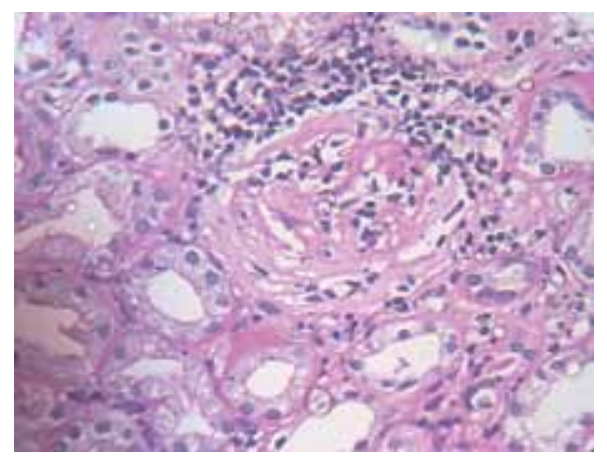

Figure 4B: ABMR. Transmural arteritis with mononuclear cell infiltration and fibrinoid changes of the media is seen (PAS stain, X400).

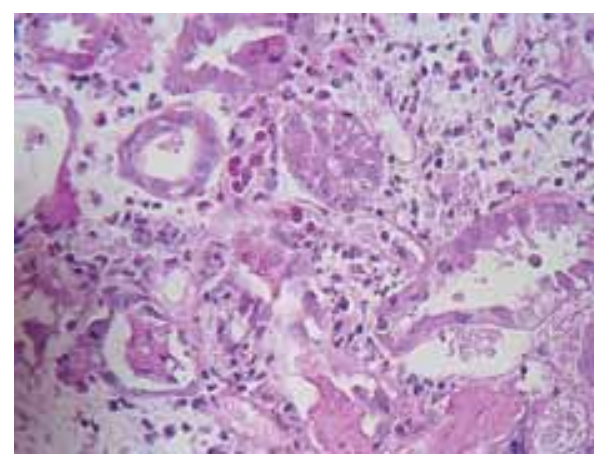

Figure 5B: Acute tubulointerstitial nephritis showing an abundant neutrophilic infiltrate and tubular micro-abscesses (PAS, stain X40). 
Differentiation from borderline changes can often be difficult. In Banff criteria ${ }^{6}$, the term "Borderline changes" is used when no intimal arteritis is present, but there are foci of tubulitis ( $\mathrm{t} 1, \mathrm{t} 2$ or $\mathrm{t} 3$ ) with minor interstitial inflammation (i0 or i1) or interstitial infiltration (i2, i3) with mild tubulitis (t1). There were 6 (6.12\%) cases of borderline lesion (fig. 2) in our study. Borderline lesions remain an unsolved problem. Some studies conclude that the most borderline cases are indeed $\mathrm{TCMR}^{20}$, but discrepant percentage $(30-80 \%)$ of borderline cases have been reported to progress to TCMR without treatment. ${ }^{21,22}$ Acknowledging such concerns, the Banff classification insists that the therapeutic decision in borderline cases must be made in the clinical context.

Interstitial inflammation with tubulitis and intimal arteritis is the hallmark of TCMR. TCMR typically develop 1-6 weeks after transplantation, but can occur at any time even after 5 years. ${ }^{3}$ Diagnosis requires a threshold level of mononuclear cell involvement of the interstitium and within tubules. The latter is best observed with a PAS stain that highlights the tubular basement membrane. On PAS stain, lymphocytes just within the non-atrophic tubular basement membrane and between epithelial cell nuclei can be seen as a dark blue nucleus, often with a clear halo, contrasting with the less dense and larger nucleus of the tubular epithelium (fig. 3A). The interstitium contains significant infiltrate of mononuclear cells involving $>25 \%$ of parenchyma with foci of moderate tubulitis (fig. 3B). Intimal arteritis refers to mononuclear cells beneath the endothelium, and preferentially affects the larger arteries. Tubulitis and arteritis are graded on the most severely affected tubule or artery, respectively. Subcapsular fibrosis and tubular atrophy with associated chronic inflammation are common in renal allograft, due to interruption of the capsular blood supply. Such changes do not represent allograft rejection.

There were 8 cases of TCMR from a week to 1 year after transplantation whereas one case of TCMR was seen at 0 -hour biopsy. The diagnosis of TCMR at 0 -hour biopsy was based on evaluating the functional deterioration of a graft. The lesion would have diagnosed as borderline lesion or subclinical rejection in biopsies without graft dysfunction.

Glomerulitis is not a recognized criterion for rejection. Collection of neutrophils within glomerular capillary loop may occasionally be seen in ABMR. Peritubular capillaritis with neutrophils, presence of anti-HLA antibodies or DSAs, acute tubular necrosis and a positive $\mathrm{C} 4 \mathrm{~d}$ stain are the usual features of ABMR. Thrombotic microangiopathy, interstitial hemorrhage, transmural arteritis and arterial fibrinoid necrosis may also be seen (fig. 4A\&B). The single risk factor for ABMR is sensitization that occurs because of previous HLA exposure from prior transplant, pregnancy or blood transfusion. There were 3 cases of ABMR developed within a week, 2 cases within a month, and one case after 2-3 months of transplantation. On the other hand, two female recipients developed ABMR after 12 months of transplantation.

ABMR usually presents in the first 3 months after transplantation. ${ }^{3}$ Because of its relatively poor prognosis, aggressive treatment with plasmapheris, mycophenolate, tacrolimus, and intravenous immunoglobulin and anti CD20 have been used. ${ }^{23}$ Documentation of DSAs and C4d staining was not always possible in our study which might have underdiagnosed the real number of cases of ABMR.

A large number of neutrophils suggest acute pyelonephritis. Bacterialacutepyelonephritis and polyomavirus nephropathy are common infections. The presence of occasional interstitial neutrophils or small collections of tubular luminal neutrophils may occur as part of damaged tubules in rejection. On the other hand, an abundant neutrophilic infiltrate or tubular micro-abscesses are unusual in rejection and would strongly suggest acute tubulointerstitial nephritis (AIN), possibly acute pyelonephritis (fig. 5A\&B). There was a case of 24 year-old male who underwent biopsies 4 times at 0 hour, $6^{\text {th }}$ day, $6^{\text {th }}$ week and $8^{\text {th }}$ week after transplantation. Biopsies persistently showed features of AIN except in implantation biopsy.

Viral infections associated with interstitial nephritis include cytomegalovirus and adenovirus. These infections are associated with distinct nuclear inclusions. There was a case of possible cytomegalovirus (CMV) infection, though it was not confirmed immunohistochemically. Most CMV infections occur in the first month after transplant. ${ }^{24}$ Active CMV infection is characterized by tubulointerstitial nephritis. Typically there is interstitial edema and plasma cell and mononuclear cell inflammatory infiltrate with tubulitis and epithelial injury. Identification of characteristic viral inclusion in tubular epithelial cells or identification of CMV antigen with immunohistochemical stains can distinguish CMV infection from acute rejection.

In the setting of a gradual rise in creatinine particularly with proteinuria, TG, chronic CNI toxicity, recurrent glomerular disease and hypertension must be considered. TG shows glomerular capillary double contours. There was only one case of TG in our study. In a study by Gloor JM et $\mathrm{al}^{25}$, TG was diagnosed in 55 patients out of 582 renal transplants. Most late allograft failure is attributed to CNI nephrotoxicity. There were 4 cases of chronic CNI toxicity in our study with prominent arteriolar hyalinosis.

Virtually all diseases that cause ESRD can recur in the allograft except Alport disease and polycystic kidney disease. Disease characteristics are similar to that in native kidneys. Although any disease that affects the native kidney can occur de novo in the allograft, those with any significant incidence include membranous glomerulonephritis and focal segmental glomerulosclerosis. De novo membranous glomerulonephritis has features similar to the disease in 
native kidneys, but shows a stronger association with hepatitis $\mathrm{C}$ virus infection. As the major glomerular disease in Nepal is membranous glomerulonephritis ${ }^{26}$, it need to be given extra emphasis while evaluating protocol or indicated renal allograft biopsies. In the absence of specific causes, one case showed massive IF/TA in 0-hour biopsy. In a study by Chalise PR et $\mathrm{al}^{1}$, the mean age of Nepalese kidney donors was $46.7 \pm 9.2$ years. One of the possibilities of IF/ TA. NOS in our study may be the age related changes of donor's kidney.

Protocol biopsies have long been a controversial issue in kidney transplantation. Relative risk of complications of the procedure must be weighed against the possible benefit and the patient must be fully informed before protocol biopsies can be obtained. Kidneys from so called "extended criteria" donors are commonly used for allografting, and these organs often have significant vascular disease, glomerulosclerosis, and/or interstitial fibrosis with tubular atrophy and loss. Indeed, it is routine to obtain a preimplantation "protocol" biopsy from most deceased donor kidneys for rapid processing to assess suitability for implantation. Baseline post implantation biopsies with optimum fixation and processing enable detection and quantification of glomerulosclerosis, small- and large-vessel chronic vascular disease, extent of fibrosis and TA and loss, any unsuspected pre-existing disease, and ischemic injury, which have prognostic implications for graft survival. ${ }^{27}$ Protocol peri-implantation biopsies, optimally done after vascular anastomosis, should be obtained as a baseline from all renal allografts. ${ }^{18}$ Biopsies should also be obtained at 1-week intervals in the context of delayed-graft function, until stable recovery of function is achieved. In sensitized patients with significant risk factors for acute rejection, a protocol biopsy should be obtained within the first 3 months. Renal allograft biopsies enable identification of active pathologic processes or emerging fibrosis in the allograft and initiation of early interventions to arrest or delay progression to overt dysfunction and later graft loss. In the future, incorporation of omics-technologies and discovery of new tissue markers with the goal of combining histopathology and molecular parameters within the Banff schema will improve histopathology-based diagnoses.

\section{CONCLUSION}

Renal allograft biopsy remains the gold standard for the diagnosis of allograft dysfunction. In the early posttransplant period (0-6 months), ABMR, acute ischemic injury and TCMR are the major differential diagnoses. Interstitial inflammation with tubulitis and intimal arteritis are diagnostic for TCMR, while ATN like lesion with positive $\mathrm{C} 4 \mathrm{~d}$ in the peritubular capillaries and transmural arteritis signify ABMR. In the late post transplant period (> 6 months after transplant), TG, Chronic CNI toxicity, viral infections and graft senescence including donor derived disease result in poor late graft survival.

\section{ACKNOWLEDGEMENTS}

The author would like to thank Raju Ranjitkar and Jeevan Pariyar of histopathology laboratory for preparing thin sections and special staining.

\section{REFERENCES}

1. Chalise PR, Shah DS, Sharma UK et al. Renal transplantation in Nepal: the first year's experience. Saudi J Kidney Dis Transpl 2010;21:559-64.

2. Dulal RK, Karki S. Nepalese kidney transplant recipient in a follow up clinic: related and unrelated living donor. J Nepal Med Assoc 2008;47:98-103.

3. D' Agati VD, Jennette JC, Silva FG. In Pathology of renal transplantation in: Donald WK, ed. Non-neoplastic kidney diseases. Washington DC: American Registry of Pathology/Armed Force Institute of Pathology; 2005:pp667-709.

4. Mao Q, Terasaki PI, Cai J et al. Extremely high association between appearance of HLA antibodies and failure of kidney grafts in a fiveyear longitudinal study. Am J Transplant 2007;7:864-71.

5. Solez $\mathrm{K}$, Axelsen RA, Benediktsson $\mathrm{H}$ et al. International standardization of criteria for the histologic diagnosis of renal allograft rejection: the Banff working classification of kidney transplant pathology. Kidney Int 1993;44:411-22.

6. Sis B, Mengel M, Haas $M$ et al. Banff ' 09 meeting report: antibody mediated graft deterioration and implementation of Banff working groups. Am J Transplant 2010;10:464-71.

7. Solez K, Colvin RB, Racusen LC et al. Banff 07 classification of renal allograft pathology: updates and future directions. Am $\mathrm{J}$ Transplant 2008;8:753-60.

8. Kikić Z, Regele H, Nordmeyer V et al. Significance of peritubular capillary, glomerular, and arteriolar C4d staining patterns in paraffin sections of early kidney transplant biopsies. Transplantation 2011;91:440-6.

9. Maeda H, Sakamoto K, Kashiwabara $\mathrm{H}$ et al. Effect of hypertension, hyperlipidemia and cyclosporine A therapy on long-term renal allograft survival. Transplant Proc 1996;28:1633-4.

10. Wavamunno MD, O'Connell PJ, Vitalone $\mathrm{M}$ et al. Transplant glomerulopathy: ultrastructural abnormalities occur early in longitudinal analysis of protocol biopsies. Am J Transplant 2007;7:2757-68.

11. Cosio FG, Gloor JM, Sethi S et al. Transplant glomerulopathy. Am J Transplant 2008;8:492-6.

12. Nankivell BJ, Chapman JR. Chronic allograft nephropathy: current concepts and future directions. Transplantation 2006;81:643-54.

13. Henderson LK, Nankivell BJ, Chapman JR. Surveillance protocol kidney transplant biopsies: their evolving role in clinical practice. Am J Transplant 2011;11:1570-5.

14. Racusen LC, Regele $\mathrm{H}$. The pathology of chronic allograft dysfunction. Kidney Int Suppl 2010;119:S27-32.

15. Rush D. Protocol biopsies for renal transplantation. Saudi J Kidney Dis Transpl 2010;21:1-9.

16. Serón D, Moreso F. Protocol biopsies in renal transplantation: prognostic value of structural monitoring. Kidney Int 2007;72:690-7.

17. Racusen LC, Solez K, Colvin RB, et al. The Banff 97 working classification of renal allograft pathology. Kidney Int 1999; I55:71323.

18. Racusen LC. Protocol Transplant Biopsies in Kidney Allografts: Why and When Are They Indicated? Clin J Am Soc Nephrol 2006;1:144-7. 
19. Dulal RK, Karki S, Dahal A. A cross sectional survey of kidney transplantation cost. J Nepal Helath Res Counc 2008;6:5-10.

20. Saad R, Gritsch HA, Sapiro R et al. Clinical significance of renal allograft biopsies with "borderline changes" as defined in the Banff Schema. Transplant 1997;64:992-5.

21. Meehan SM, Siegel CT, Aronson AJ et al. The relationship of untreated borderline infiltrates by the Banff Criteria to acute rejection in renal allograft biopsies. J Am Soc Nephrol 1999;10:1806-14.

22. Schweitzer EJ, Drachenberg CB, Anderson L et al. Significance of the Banff borderline biopsy. Am J Kidney Dis 1996;28:585-8.

23. Magil AB, Tinckam KJ. Focal peritubular capillary C4d deposition in acute rejection. Nephrol Dial Transplant 2006;21:1382-88.
24. Smith JM, McDonald RA. Renal transplantation in adolescents. Adolesc Med Clin 2005;16:201-14.

25. Gloor JM, Sethi S, Stegall MD et al. Transplant glomerulopathy: subclinical incidence and association with alloantibody. Am J Transplant 2007;7:2124-32.

26. Aryal G, Kafle RK. Hisopathological spectrum of glomerular disease in Nepal: a seven-year retrospective study. Nepal Med Coll J 2008;10:126-8.

27. Randhawa P: Role of donor kidney biopsies in renal transplantation. Transplantation 2001;71:1361-5. 